\title{
THE VISIBILITY AXIOM ON A HADAMARD MANIFOLD WHOSE GEODESIC FLOW IS OF ANOSOV TYPE
}

\author{
TETSUO UKAI
}

(Communicated by David G. Ebin)

\begin{abstract}
For a Hadamard manifold $M$, the set of points at infinity $M(\infty)$ is defined. If the geodesic flow on the unit tangent bundle of $M$ is of Anosov type, then with a certain curvature condition $M$ satisfies the Visibility Axiom. To prove this result, we use the Tits metric on $M(\infty)$.
\end{abstract}

1. Introduction. A connected, simply connected complete Riemannian manifold of nonpositive sectional curvature is called a Hadamard manifold. Throughout this paper, let $M$ denote a Hadamard manifold of dimension $n \geq 2$. Let $\langle$,$\rangle denote$ the inner product on $M$, and $d($,$) be the distance function on M$. Let $T M$ and $S M$ denote respectively the tangent bundle and the unit tangent bundle of $M$, and $\pi$ be the natural projection map onto $M$ in either case. For any vector $v \in T M$ let $\gamma_{v}$ be the unique maximal geodesic of $M$ such that $\gamma_{v}^{\prime}(0)=v$, where $\gamma_{v}^{\prime}(t)$ is the velocity of $\gamma_{v}$ at time $t$.

DEFINITION 1.1. For any $t \in R$ we define a map $T_{t}: T M \rightarrow T M$ as follows: Given a vector $v \in T M$, let $T_{t} v:=\gamma_{v}^{\prime}(t)$. The collection of maps $T_{t}$ is called the geodesic flow on $T M$.

Since the geodesic flow leaves $S M$ invariant, its restriction to $S M$ is called the geodesic flow on $S M$. If $V$ denotes the vector field on $T M$ defined by the geodesic flow, then the restriction of $V$ to $S M$ is a tangent vector field on $S M$.

DEFinition 1.2. Let $T_{t}$ be the geodesic flow on $S M$. The flow is said to be of Anosov type, if the following conditions are satisfied:

For each $v \in S M$ the tangent space $(S M)_{v}$ splits into a direct sum as follows.

$$
(S M)_{v}=X_{s}^{*}(v) \oplus X_{u}^{*}(v) \oplus Z(v)
$$

$\left(\operatorname{dim} X_{s}^{*}(v)>0, \operatorname{dim} X_{u}^{*}(v)>0, \operatorname{dim} Z(v)=1\right)$, where $Z(v)$ is generated by $V(v)$, and there exist positive numbers $a, b, c$, such that

(1) for any $\xi \in X_{s}^{*}(v)\left\|d T_{t} \xi\right\| \leq a\|\xi\| e^{-c t}$ for $t \geq 0,\left\|d T_{t} \xi\right\| \geq b\|\xi\| e^{-c t}$ for $t \leq 0$,

(2) for any $\eta \in X_{u}^{*}(v)\left\|d T_{t} \eta\right\| \leq a\|\eta\| e^{c t}$ for $t \leq 0,\left\|d T_{t} \eta\right\| \geq b\|\eta\| e^{c t}$ for $t \geq 0$, where \|\| is the natural norm on $(S M)_{v}$ defined in $\S 2$.

DEFINITION 1.3. Let $M$ be a Hadamard manifold. Two unit speed geodesics $c_{1}$, $c_{2}$ are called asymptotic, if there is a constant $b_{1}>0$, such that $d\left(c_{1}(t), c_{2}(t)\right) \leq b_{1}$ for all $t \geq 0$. The equivalence classes of this relation are called points at infinity

Received by the editors November 10, 1987.

1980 Mathematics Subject Classification (1985 Revision). Primary 53C22; Secondary 58F15, $58 \mathrm{~F} 17$.

Key words and phrases. Hadamard manifold, geodesic flow, Anosov flow, Visibility Axiom, Tits metric, Busemann function, horosphere. 
and are denoted by $M(\infty)$. For a unit speed geodesic $c: R \rightarrow M$, let $c(\infty) \in M(\infty)$ be the corresponding class, and $c(-\infty) \in M(\infty)$ the class of the reversed geodesic $t \mapsto c(-t)$.

The main purpose of this paper is to prove the following theorem.

THEOREM 4.3. Let $M$ be a Hadamard manifold whose sectional curvature $K$ satisfies $-k^{2}<K \leq 0$ for some constant $k>0$, and assume that the geodesic flow on $S M$ is of Anosov type. Then for any different points $z, w \in M(\infty)$, there exists $a$ unit speed geodesic $c$ which satisfies $c(-\infty)=z, c(\infty)=w$.

Let $p$ be a point of $M$ distinct from points $x, y \in M$. The angle subtended by $x$, $y$ at $p$ is $\measuredangle_{p}(x, y):=\measuredangle\left(\gamma_{p x}^{\prime}(0), \gamma_{p y}^{\prime}(0)\right)$, where $\gamma_{p x}, \gamma_{p y}$ are geodesics from $p$ to $x, y$ respectively.

DEFINITION 1.4. Let $M$ be a Hadamard manifold. $M$ satisfies the Visibility Axiom if given $p \in M$ and $\varepsilon>0$ there exists a number $r=r(p, \varepsilon)$ with the property:

If $\gamma:[a, b] \rightarrow M$ is a geodesic segment such that $d(p, \gamma([a, b])) \geq r$, then

$$
\measuredangle_{p}(\gamma(a), \gamma(b)) \leq \varepsilon .
$$

From Theorem 4.3, we obtain the following.

COROLlaRY 4.4. Let $M$ satisfy the hypotheses of Theorem 4.3. Then $M$ satisfies the Visibility Axiom.

REMARK 1.5. If the sectional curvature $K$ of a Hadamard manifold $M$ satisfies $K \leq c<0$ for some constant $c$, then $M$ satisfies the Visibility Axiom [7, p. 61].

I wish to thank K. Shiraiwa for his valuable advice, N. Innami and A. Katsuda for their much encouragement.

2. Anosov geodesic flows. For a manifold $X$ and $p \in X$, let $X_{p}$ denote the tangent space at $p$, and $T X$ denote the tangent bundle of $X$. For each $v \in T M$, $d \pi:(T M)_{v} \rightarrow M_{\pi(v)}$ is linear. We define a connection map $K: T(T M) \rightarrow T M$ such that for each $v \in T M, K:(T M)_{v} \rightarrow M_{\pi(v)}$ is linear in the following way. Given a vector $\xi \in(T M)_{v}$, let $Z:(-\varepsilon, \varepsilon) \rightarrow T M$ be a $C^{\infty}$ curve with $Z(0)=v$ and $Z^{\prime}(0)=\xi$. Let $\alpha=\pi \circ Z:(-\varepsilon, \varepsilon) \rightarrow M$. We define $K(\xi):=D Z / d t(0) \in M_{\pi(v)}$, where $D Z / d t(0)$ is the covariant derivative of $Z$ along $\alpha$ evaluated at $t=0 . K(\xi)$ does not depend on the curve $Z$ chosen.

One may define a natural inner product on $T M$ by the following. Given vectors $\xi, \eta \in(T M)_{v}$ let $\langle\xi, \eta\rangle_{v}:=\langle d \pi \xi, d \pi \eta\rangle_{\pi(v)}+\langle K \xi, K \eta\rangle_{\pi(v)}$. For any $v \in S M$ and any $\xi \in(S M)_{v}$, let $\|\xi\|:=\langle\xi, \xi\rangle^{1 / 2}$.

For any number $t \neq 0$, we define a linear map $\xi \mapsto \xi_{t}:(T M)_{v} \rightarrow(T M)_{v}$ for every $v \in T M$ as follows. Given a vector $v \in T M$ and a vector $\xi \in(T M)_{v}$, let $\xi_{t} \in(T M)_{v}$ be the unique vector such that $d \pi\left(\xi_{t}\right)=d \pi(\xi)$ and $d \pi \circ d T_{t}\left(\xi_{t}\right)=0$.

DEFINITION 2.1. For every $v \in S M$ let $X_{s}(v):=\left\{\xi \in(S M)_{v} \mid\langle\xi, V(v)\rangle=0\right.$ and $\xi_{t} \rightarrow \xi$ as $\left.t \rightarrow+\infty\right\}$. Let $X_{u}(v):=\left\{\xi \in(S M)_{v} \mid\langle\xi, V(v)\rangle=0\right.$ and $\xi_{t} \rightarrow \xi$ as $t \rightarrow-\infty\}$.

REMARK 2.2. For nonpositively curved manifolds one can define $X_{s}(v)$ and $X_{u}(v)$ equivalently as follows:

$$
\begin{aligned}
& X_{s}(v)=\left\{\xi \in(S M)_{v} \mid\left\|d \pi \circ d T_{t}(\xi)\right\| \leq c \text { for some } c>0 \text { and all } t \geq 0\right\} \\
& X_{u}(v)=\left\{\xi \in(S M)_{v} \mid\left\|d \pi \circ d T_{t}(\xi)\right\| \leq c \text { for some } c>0 \text { and all } t \leq 0\right\} .
\end{aligned}
$$


Proposition $2.3\left[6\right.$, p. 446]. For every $v \in S M$, both $X_{s}(v)$ and $X_{u}(v)$ are $(n-1)$-dimensional vector subspaces of $(S M)_{v}$.

For its proof, see the reference above.

If the geodesic flow on $S M$ is of Anosov type, then for each $v \in S M$ the tangent space $(S M)_{v}$ splits as Definition 1.2.

PROPOSITION 2.4 [6, p. 455]. Let $M$ be a Hadamard manifold whose sectional curvature $K$ satisfies $-k^{2}<K \leq 0$ for some constant $k>0$. Assume that the geodesic flow on $S M$ is of Anosov type. Then $X_{s}(v)=X_{s}^{*}(v)$, and $X_{u}(v)=X_{u}^{*}(v)$ for every $v \in S M$.

For its proof, see the reference above.

DEFINITION 2.5. Let $c: R \rightarrow M$ be a unit speed geodesic. For a finite point $x \in M$ the function

$$
h_{c(\infty)}(x):=\lim _{t \rightarrow \infty}\{d(x, c(t))-t\}
$$

is well defined and is called the Busemann function at $c(\infty)$. For every $t \in R$ we define $H(c(t), c(\infty)):=h_{c(\infty)}^{-1}(-t)$, which is called the horosphere through $c(t)$ with center $c(\infty)$. Since a Busemann function is of class $C^{2}[\mathbf{9}$, p. 484], a horosphere is a $C^{2}$-submanifold of $M$.

DEFinition 2.6. For $v \in S M$, take the geodesic $c: R \rightarrow M$ such that $c^{\prime}(0)=v$. Let

and

$$
\mathscr{H}^{+}(v):=\left\{\left(p,-\operatorname{grad} h_{c(\infty)}(p)\right) \in S M \mid p \in H(c(0), c(\infty))\right\}
$$

$$
\mathscr{H}^{-}(v):=\left\{\left(p, \operatorname{grad} h_{c(-\infty)}(p)\right) \in S M \mid p \in H(c(0), c(-\infty))\right\},
$$

where $\operatorname{grad} h_{c(\infty)}$ is the gradient vector field of $h_{c(\infty)}$. Then $\mathscr{H}^{+}(v)$ and $\mathscr{H}^{-}(v)$ are $C^{2}$-submanifolds of $S M$ with dimension $n-1$.

LEMMA 2.7. Let $v \in S M$. Then $X_{s}(w)=\left(\mathscr{H}^{+}(v)\right)_{w}$ for $w \in \mathscr{H}^{+}(v)$, and $X_{u}(w)=\left(\mathscr{H}^{-}(v)\right)_{w}$ for $w \in \mathscr{H}^{-}(v)$.

ProOF. Since $\mathscr{H}^{+}(w)=\mathscr{H}^{+}(v)$ for any $w \in \mathscr{H}^{+}(v)$, we prove this lemma only when $w=v$. Let $c: R \rightarrow M$ be the unique geodesic with $c^{\prime}(0)=v$. Take $u \in M_{\pi(v)}$, such that $u \perp v$. Let $a:(-\varepsilon, \varepsilon) \rightarrow H(c(0), c(\infty))$ be a curve with $a^{\prime}(0)=u$, and for $n \rightarrow \infty a_{n}:(-\varepsilon, \varepsilon) \rightarrow S_{n}(c(n))$ be curves with $a_{n}^{\prime}(0)=u$, where $S_{n}(c(n)):=$ $\{p \in M \mid d(p, c(n))=n\}$. For $s \in(-\varepsilon, \varepsilon)$, let $\gamma_{s}$ and $\gamma_{n, s}$ be the unique unit speed geodesics from $a(s)$ to $c(\infty)$ and from $a_{n}(s)$ to $c(n)$ respectively. We define variations $b, b_{n}:(-\varepsilon, \varepsilon) \times(-\varepsilon, \varepsilon) \rightarrow M$ by $b(s, t):=\gamma_{s}(t)$ and $b_{n}(s, t):=\gamma_{n, s}(t)$ respectively. Let $Y:=-\operatorname{grad} h_{c(\infty)}$ and $Y_{n}:=-\operatorname{grad} d_{c(n)}$, where $d_{c(n)}: p \mapsto$ $d(c(n), p)$. Then for each $(s, t) \in(-\varepsilon, \varepsilon) \times(-\varepsilon, \varepsilon)$, we have $Y(b(s, t))=\gamma_{s}^{\prime}(t)$, and $Y_{n}\left(b_{n}(s, t)\right)=\gamma_{n, s}^{\prime}(t)$. Then it is known that $\nabla_{u} Y_{n}(c(0)) \rightarrow \nabla_{u} Y(c(0))$ as $n \rightarrow \infty$ [9, p. 484], where $\nabla_{u} Y$ is the covariant derivative of $Y$ in the direction $u$. Let $\xi:=\left(u, \nabla_{u} Y(c(0))\right) \in(S M)_{v}$, where $d \pi \xi=u$ and $K \xi=\nabla_{u} Y(c(0))$. Then

$$
\xi=\left(\frac{\partial b}{\partial s}(0,0), \frac{D}{\partial s} \frac{\partial b}{\partial t}(0,0)\right)
$$

and

$$
\xi_{n}=\left(d \pi\left(\xi_{n}\right), K\left(\xi_{n}\right)\right)=\left(u, \frac{D}{\partial s} \frac{\partial b_{n}}{\partial t}(0,0)\right)=\left(u, \nabla_{u} Y_{n}(c(0))\right)
$$


[6, p. 441]. So $\xi \in\left(\mathscr{H}^{+}(v)\right)_{v}$ and $\xi_{n} \rightarrow \xi$ as $n \rightarrow \infty$. It proves that $\left(\mathscr{H}^{+}(v)\right)_{v} \subset$ $X_{s}(v)$. Since both of these spaces have the same dimension $n-1$ (Proposition 2.3), we see that $\left(\mathscr{H}^{+}(v)\right)_{v}=X_{s}(v)$. The second part of this lemma is proved similarly.

LEMMA 2.8. Let $M$ be a Hadamard manifold whose sectional curvature $K$ satisfies $-k^{2}<K \leq 0$ for some constant $k>0$. Assume that the geodesic flow on $S M$ is of Anosov type, and let $c_{1}, c_{2}: R \rightarrow M$ be unit speed geodesics such that $c_{1}(-\infty)=c_{2}(-\infty)$ and $c_{1}(t), c_{2}(t)$ lie on the same horosphere with center $c_{1}(-\infty)$ for any $t \in R$. Then for every $t \geq 0$,

$$
d^{H}\left(c_{1}(t), c_{2}(t)\right) \geq b\left(1+k^{2}\right)^{-1 / 2} e^{c t} d^{H}\left(c_{1}(0), c_{2}(0)\right)
$$

where $d^{H}$ denotes the distance function in the corresponding horosphere.

PROOF. Since horospheres are complete $C^{2}$ submanifolds of $M$, for any $t \geq 0$ there exists a geodesic $\gamma_{t}:[0,1] \rightarrow H\left(c_{1}(t), c_{1}(-\infty)\right)$ in $H\left(c_{1}(t), c_{1}(-\infty)\right)$ such that $\gamma_{t}(0)=c_{1}(t), \gamma_{t}(1)=c_{2}(t)$ and $L\left(\gamma_{t}\right)=d^{H}\left(c_{1}(t), c_{2}(t)\right)$, where $L()$ denotes the length of a curve. For a fixed number $t \geq 0$, we define a curve $\mu:[0,1] \rightarrow$ $H\left(c_{1}(0), c_{1}(-\infty)\right)$ by $\mu(s):=\pi \circ T_{t}\left(-\operatorname{grad} h_{c_{1}(-\infty)}\left(\gamma_{t}(s)\right)\right)$. Consider a curve $W:[0,1] \rightarrow S M$ defined by $W(s):=\left(\mu(s),-\operatorname{grad} h_{c_{1}(-\infty)}(\mu(s))\right)$, and let $\xi(s):=$ $W^{\prime}(s) \in(S M)_{W(s)}$. Then from Lemma 2.7 and Proposition 2.4, we have $\xi(s) \in$ $X_{s}(W(s))=X_{s}^{*}(W(s))$ for any $s \in[0,1]$. Since the sectional curvature $K$ satisfies $-k^{2}<K \leq 0$, for any $v \in S M$ and any $\xi \in X_{s}(v)$ or $X_{u}(v)$ we have $\|K \xi\| \leq k\|d \pi \xi\|$ [6, p. 448]. Now the Anosov condition implies

$$
\left\|d T_{-t} \xi(s)\right\| \geq b\|\xi(s)\| e^{c t} \quad \text { for } t \geq 0 .
$$

Since $d T_{-t} \xi(s) \in X_{s}\left(-\operatorname{grad} h_{c_{1}(-\infty)}\left(\gamma_{t}(s)\right)\right)$, it follows that

$$
\begin{aligned}
\left(1+k^{2}\right)^{1 / 2}\left\|d \pi \circ d T_{-t} \xi(s)\right\| & \geq\left(\left\|d \pi \circ d T_{-t} \xi(s)\right\|^{2}+\left\|K \circ d T_{-t} \xi(s)\right\|^{2}\right)^{1 / 2} \\
& =\left\|d T_{-t} \xi(s)\right\| \geq b\|\xi(s)\| e^{c t} \geq b\|d \pi \xi(s)\| e^{c t} .
\end{aligned}
$$

For $s \in[0,1], \gamma_{t}^{\prime}(s)=d \pi \circ d T_{-t} \xi(s)$ and $\mu^{\prime}(s)=d \pi \xi(s)$. So we have,

$$
\begin{aligned}
L\left(\gamma_{t}\right) & =\int_{0}^{1}\left\|\gamma_{t}^{\prime}(s)\right\| d s \geq b\left(1+k^{2}\right)^{-1 / 2} e^{c t} \int_{0}^{1}\left\|\mu^{\prime}(s)\right\| d s \\
& =b\left(1+k^{2}\right)^{-1 / 2} e^{c t} L(\mu) .
\end{aligned}
$$

Now this lemma follows from the fact $d^{H}\left(c_{1}(t), c_{2}(t)\right)=L\left(\gamma_{t}\right)$ and $d^{H}\left(c_{1}(0), c_{2}(0)\right)$ $=L\left(\gamma_{0}\right) \leq L(\mu)$.

DEFINITION 2.9. Let $\gamma$ be a geodesic of $M$. A vector field $Y$ along $\gamma$ is a Jacobi field if $(D / d t)(D Y / d t)+R\left(\gamma^{\prime}, Y\right) \gamma^{\prime}=0$, where $\gamma^{\prime}$ is the velocity vector field of $\gamma$, and $R$ is the curvature tensor of $M$.

PROPOSITION 2.10 [6, p. 455]. Let $M$ be a Hadamard manifold whose sectional curvature $K$ satisfies $-k^{2}<K \leq 0$ for some constant $k>0$, and assume that the geodesic flow on $S M$ is of Anosov type. Then there exists no nonzero perpendicular Jacobi field $Y$ along a unit speed geodesic $\gamma$ of $M$ such that $\|Y(t)\|$ is bounded above for all $t \in R$.

For its proof, see the reference above. 
3. The Tits metric. In this section, every lemma is related with the Tits metric. Here its proof is omitted (see $[3, \S 4]$ ).

DEFINITION 3.1. Let $M$ be a Hadamard manifold. Fix a point $x \in M$, and for any $z, w \in M(\infty)$ let $c_{1}, c_{2}$ be the unique unit speed geodesics from $x$ to $z$, from $x$ to $w$ respectively. Then $t \mapsto(1 / t) d^{S}\left(c_{1}(t), c_{2}(t)\right)$ is increasing, where $d^{S}$ denotes the distance function in the corresponding sphere $S_{t}(x):=\{y \in M \mid d(x, y)=t\}$. We define the Tits metric on $M(\infty)$ by

$$
\operatorname{Td}(z, w):=\lim _{t \rightarrow \infty}(1 / t) d^{S}\left(c_{1}(t), c_{2}(t)\right)
$$

(cf. [3, p. 43]).

REMARK 3.2. The definition of the Tits metric does not depend on $x \in M$ chosen. $T d: M(\infty) \times M(\infty) \rightarrow[0, \infty) \cup\{\infty\}$ is a metric (i.e. $d(z, w)=0 \Leftrightarrow z=w$, $d(z, w)=d(w, z), d(z, v)+d(v, w) \geq d(z, w))$. We allow that points have infinite distance.

LEMMA 3.3 [3, p. 46]. Let $z, w \in M(\infty)$. If $T d(z, w)>\pi$, then there exists a unit speed geodesic $c: R \rightarrow M$ such that $c(-\infty)=z, c(\infty)=w$.

LEMMA 3.4 [3, p. 46]. If $c: R \rightarrow M$ is a unit speed geodesic, then

$$
\operatorname{Td}(c(-\infty), c(\infty)) \geq \pi \text {. }
$$

Equality holds if and only if $c$ bounds a totally geodesic flat half plane.

DEFINITION 3.5. Let $c:[0,1] \rightarrow M(\infty)$ be a continuous curve in the Titstopology (i.e. the topology defined by the Tits metric). We define the length of $c$ by $L(c):=\sup \sum_{i=0}^{m-1} T d\left(c\left(t_{i}\right), c\left(t_{i+1}\right)\right)$, where $0=t_{0} \leq t_{1} \leq \cdots \leq t_{m}=1$ is a subdivision of $[0,1]$ and the supremum is taken over all subdivisions.

LEMMA 3.6 [3, p. 49]. Let $z, w \in M(\infty)$. If $T d(z, w)<\infty$, then there exists a continuous curve $h:[0,1] \rightarrow M(\infty)$ in the Tits-topology such that $h(0)=z$, $h(1)=w$ and $L\left(\left.h\right|_{[0, t]}\right)=t T d(z, w)$ for any $t \in[0,1] . \quad$ ( $h$ is called a minimal geodesic in $M(\infty)$ from $z$ to $w$.)

\section{The Visibility Axiom.}

LEMMA 4.1 [3, p. 54]. Let $M$ be a Hadamard manifold. $M$ satisfies the Visibility Axiom if and only if for every different points $z, w \in M(\infty)$ there is a unit speed geodesic $c: R \rightarrow M$ with $c(-\infty)=z, c(\infty)=w$.

For its proof, see the reference above.

PROPOSITION $4.2[9$, p. 488]. Let $M$ be a Hadamard manifold whose sectional curvature $K$ satisfies $-k^{2} \leq K \leq 0$ for some constant $k>0$. Let $H$ be an arbitrary horosphere of $M$. Then for any points $p, q \in H$ we have

$$
d^{H}(p, q) \leq \frac{2}{k} \sinh \frac{k}{2} d(p, q)
$$

where $d^{H}$ is the distance function in the corresponding horosphere.

For its proof, see the reference above. 
THEOREM 4.3. Let $M$ be a Hadamard manifold whose sectional curvature $K$ satisfies $-k^{2}<K \leq 0$ for some constant $k>0$, and assume that the geodesic flow on $S M$ is of Anosov type. Then for any different points $z, w \in M(\infty)$, there exists $a$ unit speed geodesic $c$ which satisfies $c(-\infty)=z, c(\infty)=w$.

PROOF. For any different points $z, w \in M(\infty)$, we prove $T d(z, w)=\infty$. Then this theorem follows from Lemma 3.3. Assume that $T d(z, w)<\infty$ for some $z, w \in$ $M(\infty), z \neq w$. From Lemma 3.6, there exists a minimal geodesic $h:[0,1] \rightarrow M(\infty)$ from $z$ to $w$. Let $\gamma: R \rightarrow M$ be a unit speed geodesic with $\gamma(\infty)=z$, and let $a_{1}:=T d(\gamma(-\infty), \gamma(\infty))$. From Lemma 3.4, $a_{1} \geq \pi$. If $a_{1}=\pi$, then again from Lemma $3.4 \gamma$ bounds a totally geodesic flat half plane. Then using the variation of $\gamma$ through geodesics, we obtain a nonzero perpendicular Jacobi field $Y$ along $\gamma$ such that $\|Y(t)\|=1$ for all $t \in R$. This contradicts Proposition 2.10, and it follows that $a_{1}>\pi$. Since $h(0)=z$, there exists a number $s \in(0,1)$ with

$$
\operatorname{Td}(z, h(s))<\min \left\{2 c / k, a_{1}-\pi\right\} .
$$

Let $v:=h(s)$. Since $T d(\gamma(-\infty), v) \geq T d(\gamma(-\infty), \gamma(\infty))-T d(\gamma(\infty), v)>a_{1}-\left(a_{1}-\right.$ $\pi)=\pi$, from Lemma 3.3 there exists a unit speed geodesic $\mu: R \rightarrow M$ such that $\mu(-\infty)=\gamma(-\infty), \mu(\infty)=v$ and $\gamma(t), \mu(t)$ lie on the same horosphere with center $\gamma(-\infty)$ for any $t \in R$. Let $\mu_{0}:[0, \infty) \rightarrow M$ be the unique unit speed geodesic with $\mu_{0}(0)=\gamma(0), \mu_{0}(\infty)=v$. Since $\mu$ and $\mu_{0}$ are asymptotic, there exists a number $b_{1}>0$ such that

$$
d\left(\mu(t), \mu_{0}(t)\right) \leq b_{1} \quad \text { for any } t \geq 0 .
$$

From Lemma 2.8 and Proposition 4.2, for any $t \geq 0$

$$
\begin{aligned}
b\left(1+k^{2}\right)^{-1 / 2} e^{c t} d^{H}(\gamma(0), \mu(0)) & \leq d^{H}(\gamma(t), \mu(t)) \\
& \leq(2 / k) \sinh ((k / 2) d(\gamma(t), \mu(t))) \\
& <(1 / k) \exp ((k / 2) d(\gamma(t), \mu(t))) .
\end{aligned}
$$

Then,

$$
b k\left(1+k^{2}\right)^{-1 / 2} e^{c t} d^{H}(\gamma(0), \mu(0))<\exp ((k / 2) d(\gamma(t), \mu(t))) .
$$

Take the logarithm of both sides and divide by $k t / 2$ to obtain

$$
2 c / k+(2 / k t) B<(1 / t) d(\gamma(t), \mu(t)),
$$

where $B=\log \left\{b k\left(1+k^{2}\right)^{-1 / 2} d^{H}(\gamma(0), \mu(0))\right\}$.

Then for any $\varepsilon>0$ there exists a number $N>0$ such that $2 c / k-\varepsilon / 2<$ $(1 / t) d(\gamma(t), \mu(t))$ for any $t \geq N$. Choose a number $t_{1} \geq N$ such that $b_{1} / t_{1}<\varepsilon / 2$. From $(* *)$, we have

$$
\begin{aligned}
T d(z, v) & =\lim _{t \rightarrow \infty}(1 / t) d^{S}\left(\gamma(t), \mu_{0}(t)\right) \\
& \geq\left(1 / t_{1}\right) d^{S}\left(\gamma\left(t_{1}\right), \mu_{0}\left(t_{1}\right)\right) \\
& \geq\left(1 / t_{1}\right) d\left(\gamma\left(t_{1}\right), \mu_{0}\left(t_{1}\right)\right) \\
& \geq\left(1 / t_{1}\right)\left\{d\left(\gamma\left(t_{1}\right), \mu\left(t_{1}\right)\right)-b_{1}\right\} \\
& >(2 c / k-\varepsilon / 2)-\varepsilon / 2=2 c / k-\varepsilon .
\end{aligned}
$$

Since $\varepsilon>0$ is arbitrary, we have $T d(z, v) \geq 2 c / k$. But this contradicts $(*)$. 
COROLLARY 4.4. Let $M$ satisfy the hypotheses of Theorem 4.3. Then $M$ satisfies the Visibility Axiom.

PROOF. This corollary follows from Theorem 4.3 and Lemma 4.1.

\section{REFERENCES}

1. D. V. Anosov, Geodesic flows on closed Riemann manifolds with negative curvature, Proc. Steklov Inst. Math. 90 (1967).

2. V. I. Arnold and A. Avez, Ergodic problems of classical mechanics, Benjamin, New York and Amsterdam, 1968.

3. W. Ballmann, M. Gromov and V. Schroeder, Manifolds of nonpositive curvature, Birkhäuser, Boston, Mass., 1985.

4. R. L. Bishop and R. J. Crittenden, Geometry of manifolds, Academic Press, New York, 1964.

5. J. Cheeger and D. G. Ebin, Comparison theorems in Riemannian geometry, North-Holland, Amsterdam, 1975.

6. P. Eberlein, When is a geodesic flow of Anosov type? I, J. Differential Geometry 8 (1973), 437-463.

7. P. Eberlein and B. O'Neill, Visibility manifolds, Pacific J. Math. 46 (1973), 45-110.

8. J. H. Eschenburg, Horospheres and the stable part of the geodesic flow, Math. Z. 153 (1977), 237-251.

9. E. Heintze and H. C. Im Hof, Geometry of horospheres, J. Differential Geometry 12 (1977), 481-491.

10. J. Milnor, Morse theory, Princeton Univ. Press, Princeton, N. J., 1969.

Department of Mathematics, Faculty of Science, Nagoya University, CHIKUSA-KU, NAGOYA 464, JAPAN 